\title{
CORRELATION OF THE IGG INDEX AND OLIGOCLONAL BANDS IN THE CEREBROSPINAL FLUID OF PATIENTS WITH MULTIPLE SCLEROSIS
}

\author{
Jan Mares ${ }^{\mathrm{a} *}$, Roman Herziga, Karel Urbanek ${ }^{\mathrm{a}}$, Vladimira Sladkova ${ }^{\mathrm{a}}$, Jaroslava Sklenarova ${ }^{\mathrm{b}}$, \\ Vojtech Bekarek $^{\mathrm{b}}$, Petr Schneiderka ${ }^{\mathrm{b}}$, Jana Zapletalova ${ }^{\mathrm{c}}$, Petr Kanovsky ${ }^{\mathrm{a}}$
}

\author{
a Department of Neurology, University Hospital, Olomouc, Czech Republic \\ ${ }^{b}$ Department of Clinical Biochemistry, University Hospital, Olomouc \\ c Institute of Biophysics, Palacký University, Olomouc \\ e-mail:maresja@seznam.cz
}

Received: July 22, 2008; Accepted (with revision): December 11, 2008

Key words: Oligoclonal bands/Cerebrospinal fluid/IgG index/Multiple sclerosis

Aims: The aim of this study was to assess the correlation between $\mathrm{IgG}$ index values and the number of the oligoclonal IgG bands (OCB) in the cerebrospinal fluid (CSF) of patients with multiple sclerosis (MS).

Material and Methods: The set of 150 patients consisted of 41 males and 109 females (aged 18-68, mean 36.6 \pm 10.1 years). The CSF collected by a lumbar puncture was examined evaluating intrathecal synthesis using the IgG index and determining OCB. The number of alkaline OCB in the CSF was assessed using the method of isoelectric focusing. Pearson's correlation analysis, and homogeneity $\chi^{2}$ test, Mann-Whitney test, paired-sample t-test (parametric) and Wilcoxon signed-ranks test (nonparametric) were used to evaluate the statistical significance of the results.

Results: No positive correlation between the IgG index and the number of OCB was found. Mann-Whitney test also failed to demonstrate any significant difference of the IgG index values in patients both with the OCB number $\geq 2$ and $<2$.

Conclusion: This study did not confirm any correlation between the IgG index values and the OCB number in the CSF of MS patients.

\section{INTRODUCTION}

Immunoglobulins are glycoproteins produced by plasmocytes. In contrast to the known and characteristic reaction in the serum, where antibody production switches from the IgM to IgG class in the subacute and chronic phase of the disease, such a transition is not present in the intrathecal synthesis. The characteristic immunoglobulin pattern in classes IgG, IgM and IgA existing in the central nervous system (CNS) remains relatively constant from the disease onset. Intrathecal IgG synthesis represents local IgG production within the CNS compartment, originates from perivascular infiltrates of $\mathrm{B}$ lymphocytes. Quantitative assessment of the intrathecal IgG synthesis is based on the relationship between IgG and albumin concentrations in the CSF and serum. Oligoclonal IgG immunoglobulins represent a primarily polyspecific and only subsequently an oligoclonal type of immune response. This produces an extensive spectrum of non-specific antibodies of "nonsense" nature.

In many countries, information obtained from detailed assessment of the CSF is currently important in the MS diagnostics. In all MS stages, increased IgG levels, which after correction for the function of the blood-brain barrier show intrathecal synthesis, can be found. It is possible to demonstrate this as OCB by which the immunoglobulins participating in the destruction of the myelin covering of axons line up to special patterns. OCB assessment is the most specific CSF test for MS diagnosis ${ }^{1}$ - their presence supports this diagnosis as they are not found in the serum of these patients and are therefore a proof of antibody production directly in the CNS. OCB are present (as 2 or more) in the CSF of $95-100 \%$ of MS patients ${ }^{2,3}$. In $40 \%$ of MS patients, OCB may be found in the serum as well. OCB can be present even in the case, when the CSF IgG level is normal. Finally, these bands are not specific to for MS - they are also found in various inflammatory disorders as well as in chronic CNS infections. On the other hand, they are not present in neurodegenerative diseases, acute disseminated encephalomyelitis, Guillain-Barré syndrome, toxic, metabolic, traumatic or psychiatric disorders, radicular syndromes or in the majority of peripheral neuropathies ${ }^{4}$. OCB have a predictive value in the case of a negative magnetic resonance imaging finding (MRI), but there is no direct correlation between the OCB in the CSF and the demyelinating process as assessed by the MRI.

Treatment for the relapsing-remitting MS with betainterferons and glatiramer acetate leads to significant decrease in the number of relapses and to the shortening of their duration as well as to a slower worsening in the MRI findings. Only limited data on the immunological CSF findings during the disease-modifying drug (DMD) therapy has been published in the current literature and the changes in OCB patterns have mostly not been described $^{5}$.

The aim of this study was to assess the correlation between IgG index values and the OCB number in the CSF of patients with MS. 
Table 1. IgG Index values.

\begin{tabular}{|c|c|c|c|}
\hline IgG Index & Frequency & Percent & Valid Percent \\
\hline$<=0.7$ & 91 & 60.7 & 61.9 \\
\hline$>0.7$ & 56 & 37.3 & 38.1 \\
\hline Total & 147 & 98.0 & 100.0 \\
\hline Unlisted & 3 & 2.0 & \\
\hline Total & 150 & 100.0 & \\
\hline
\end{tabular}

Table 2. Number of oligoclonal IgG bands.

\begin{tabular}{|c|c|c|c|}
\hline IgG Index & Frequency & Percent & Valid Percent \\
\hline$>=2$ & 97 & 64.7 & 70.3 \\
\hline$<2$ & 41 & 27.3 & 29.7 \\
\hline Total & 138 & 92.0 & 100.0 \\
\hline Unlisted & 12 & 8.0 & \\
\hline Total & 150 & 100.0 & \\
\hline
\end{tabular}

\section{MATERIAL AND METHODS}

The sample of 150 MS patients consisted of 41 males and 109 females (aged 18-68, mean $36.6 \pm 10.1$ years). The diagnosis of RRMS was established based on the McDonald's criteria. All patients were treated at the Department of Neurology, University Hospital, Olomouc, Czech Republic, between 2001 and 2005.

In all patients, the CSF was collected by routine lumbar puncture as part of the standard diagnostic process. The assessment of the IgG index and of the OCB was used for the evaluation of the intrathecal synthesis. IgG method of Pharmacia Biotech (Uppsala, Sweden) modified for using of acrylamid gel PhastGel ICF 3-9 and by isoelectric focusing (IEF) $\left(\right.$ ref. $^{6}$ ) with successive affinity immunoblot was used. The number of OCB in the CSF was assessed by the method of isoelectric focusation (IEF). The samples were examined in a laboratory with an international certificate for the IEF method (KB/0079).

Pearson's correlation analysis, and homogeneity $\chi^{2}$ test, Mann-Whitney test, paired-sample t-test (parametric) and Wilcoxon signed-ranks test (nonparametric) were used to evaluate the statistical significance of the results, using SPSS-10 software package (SPSS, Chicago, USA). Sensitivity and specificity of the $\operatorname{IgG}$ index and the OCB number for the prediction of MS diagnosis were also assessed.

The study was conducted in accordance with the Helsinki Declaration of 1975 (as revised in 1983) and it was approved by local ethics committee of University Hospital in Olomouc, Czech Republic.

\section{RESULTS}

Only OCB present in the CSF and absent from serum were considered. IgG index and the OCB number are summarized in the Tables 1 and 2.

No positive correlation between the $\operatorname{IgG}$ index and the number of OCB was found by the Pearson's correlation and nonparametric Spearman correlation. Using the Mann-Whitney also test did not demonstrate any significant difference of the $\mathrm{IgG}$ index values in patients both with the OCB number $\geq 2$ and $<2$.

We also determined the sensitivity and the specificity of the technique used in our laboratory: characteristics of diagnostic test of IgG index $>0.7$ for prediction of MS diagnosis: sensitivity - $38.1 \%$, specificity $-91.7 \%$, accuracy $-77.6 \%$, positive predictive value $-62.2 \%$, negative predictive value $-80.5 \%$. Characteristics of the diagnostic test of OCB number $>=2$ for prediction of MS diagnosis: sensitivity $-70.3 \%$, specificity $-76.1 \%$, accuracy $-74.4 \%$, positive predictive value $-53.3 \%$, negative predictive value $-86.8 \%$. Characteristics of the diagnostic test both of of $\operatorname{IgG}$ index $>0.7$ and oligoclonal IgG bands number $>=2$ for prediction of MS diagnosis: sensitivity $-77.0 \%$, specificity $-73.7 \%$, accuracy $-74.6 \%$, positive predictive value $-51.1 \%$, negative predictive value $-90.0 \%$.

\section{DISCUSSION}

The dynamics of the appearance and the development of OCB during the course of MS is not yet generally known ${ }^{7}$. In the study performed by Kaiser et al. ${ }^{8}$, only two cases $(1 \%)$ out of the 185 CSF samples obtained from MS patients demonstrated specificity of OCB antibodies against known CNS antigens. Rudick et al. ${ }^{5}$ found 


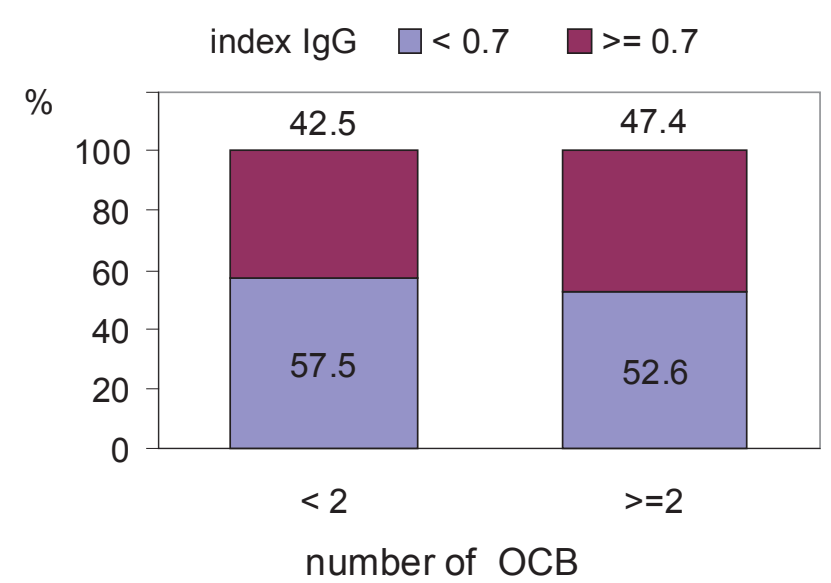

Fig. 1. Correlation of the $\operatorname{IgG}$ index a OCB number

no changes in the $\mathrm{IgG}$ index, light kappa chains or OCB patterns in 137 repeated CSF samples and 2 years after the beginning of Avonex therapy. Kinnunen et al..$^{9}$ found that in $3(50 \%)$ out of the 6 patients with progressive MS, alfa-interferon therapy lead to increased intrathecal synthesis and production of OCB. Livrea et al..$^{10}$ found no correlation between the OCB pattern or amounts and age, duration, clinical course or therapy of the disease.

Concerning to correlation between the IgG index and OCB there is a lack of data in the current literature Kaiser et al. ${ }^{11}$ used two quantitative methods of determining the intrathecal synthesis of IgG for their usefulness in deciding about the necessity of further investigations of OCB in the CSF. While OCB could be detected in no patient with an IgG index <0.45, OCB were always demonstrated in patients with an index $>0.80$. Even though a range of $0.45-0.8$ OCB was only detected in 268 out of 1316 patients (20.4\%), in 190 out of 268 samples (70.8\%) OCB were the only criterion for intrathecal synthesis of IgG. OCB were always detected if local production of IgG was $>12 \%$. In Anderson ${ }^{12}$ et al. study, CSF IgG index fell significantly with initial methylprednisolone treatment of $26 \mathrm{MS}$ patients and significant reductions in CSF IgG, CSF albumin, serum IgG and serum albumin levels and CSF IgG synthesis rate in the first and repeat treatment groups were described. Mayringer et al. ${ }^{13}$ investigated if there is a correlation between the frequency of OCB and the $\mathrm{IgG}$ index and if the $\mathrm{IgG}$ index predicts the diagnosis of a demyelinating CNS disease. They found a positive correlation between the $\mathrm{IgG}$ index and the frequency of $\mathrm{OCB}$ as well as the probability of demyelinating CNS disease.

In contrast we found no positive correlation between both parameters of intrathecal synthesis. In accordance with our findings, Rochelli et al..$^{14}$ reported 63 out of 70 patients with definite MS and 24 out of 35 with probable MS had oligoclonal bands in the CSF and in the 18 patients with normal OCB pattern no statistically significant difference was found in the quantitative CSF parameters (IgG index, IgG synthesis and serum/CSF albumin quotient) compared to the patients with the OCB in CSF.
Also in Poloni ${ }^{15}$, in a group of 120 MS patients a group of 20 patients with normal IgG profile were compared with a group of 22 patients selected by random out of 100 with IgG oligoclonal bands and no statistically significant difference was discovered between these groups.

\section{CONCLUSION}

This study found no correlation between the $\operatorname{IgG}$ index values and the OCB count in the CSF of MS patients. Further studies are needed to clarify the issue.

\section{REFERENCES}

1. Andersson M, Alvarez-Carmeno J, Bernardi G, et al. Cerebrospinal fluid in the diagnosis of multiple sclerosis: A consensus report. J Neurol Neurosurg Psychiatry 1994; 57:897-902.

2. Caudie C, Allauzen O, Bancel J, Later R. Diagnostic usefulness of oligoclonal immunoglobulin $\mathrm{G}$ bands in cerebro-spinal fluid using isoelectric focusing in early diagnosis of multiple sclerosis. Annales de Biologie Clinique 2002; 58:187-93.

3. Miller JR, Burke AM, Bever CT. Occurrence of oligoclonal bands in multiple sclerosis and other CNS diseases. Ann Neurol Jan 1983; 13(1):53-8.

4. Sindic CHJM, Van Antwerpen MP, Goffette S. The Intrathecal Humoral Immune Response: Laboratory Analysis and Clinical Relevance. Clin Chem Lab Med 2001; 39(4): 333-340.

5. Rudick RA, Cookfair DL, Simonian NA, Ransohoff RM, Richert JR, Jacobs LD, el al. Cerebrospinal fluid abnormalities in a phase III trial of Avonex (IFNbeta-1a) for relapsing multiple sclerosis. The Multiple Sclerosis Collaborative Research Group. J Neuroimmunol 1999; 93(1-2):8-14.

6. Correale J, de los Milagros Bassani Molinas M. Oligoclonal bands and antibody responses in multiple sclerosis. J Neurol 2002; 249:375-89.

7. Antel JP, Birnbaum G, Hartung HP (1998) Clinical neuroimunology. Blackwell Science. London, 1998; 423

8. Kaiser R, Obert M, Kaufmann R, Czygan M. IgG-antibodies to CNS proteins in patients with multiple sclerosis. Eur J Med Res 1997; 2(4):169-72.

9. Kinnunen E, Timonen T, Pirttila T, Kalliomaki P, Ketonen L, Matikainen E, et al. Effects of recombinant alpha-2b-interferon therapy in patients with progressive MS. Acta Neurol Scand 1993; 87(6):457-60.

10. Livrea P, Trojano M, Simone IL, Zimatore GB, Lamontanara G, Leante R. Intrathecal IgG synthesis in multiple sclerosis: comparison between isoelectric focusing and quantitative estimation of cerebrospinal fluid IgG. J Neurol. 1981; 224(3):159-69.

11. Kaiser R, Czygan M, Kaufmann R, Lucking CH. Intrathecal IgG synthesis: when is determination of oligoclonal bands necessary? Nervenarzt. 1995; 66(8):618-23.

12. Anderson TJ, Donaldson IM, Sheat JM, George PM. Methylprednisolone in multiple sclerosis exacerbation: changes in CSF parameters. Aust N Z J Med 1990; 20(6):794-7.

13. Mayringer I, Timeltaler B, Deisenhammer F. Correlation between the IgG index, oligoclonal bands in CSF, and the diagnosis of demyelinating diseases. Eur J Neurol. 2005; 12(7):527-30.

14. Rocchelli B, Poloni M, Mazzarello P, Delodovici M. Clinical and CSF findings in multiple sclerosis patients with or without IgG oligoclonal bands at isoelectric focusing examination of CSF and serum proteins. Eur Neurol. 1983; 22(1):35-42.

15. Poloni M, Rocchelli B, Mazzarello P, Delodovici M. Relation between the clinical and cerebrospinal fluid parameters in multiple sclerosis, with special reference to subjects without oligoclonal IgG in the cerebrospinal fluid. Riv Neurol. 1981; 51(2):69-80. 
